\title{
Factors associated with prevalence, awareness, treatment and control of hypertension in urban adults from 33 communities in China: the CHPSNE Study
}

\author{
Shen Tian ${ }^{1}$, Guang-Hui Dong ${ }^{2}$, Da Wang ${ }^{2}$, Miao-Miao Liu ${ }^{2}$, Qiao Lin ${ }^{1}$, Xiu-Jun Meng ${ }^{1}$, Li-Xia Xu ${ }^{1}$, \\ Hua Hou ${ }^{1}$ and Yu-Feng Ren ${ }^{1}$
}

\begin{abstract}
Using a randomized multistage cluster sampling, we studied the factors associated with the prevalence, awareness, treatment and control of hypertension (defined as systolic and diastolic blood pressure (BP) measurements $\geqslant 140$ and $90 \mathrm{~mm} \mathrm{Hg}$, respectively, or current drug treatment for hypertension) in a representative sample of the urban Chinese population. The participants were 18-74 years of age and had lived for at least 5 years in an area comprising 33 communities in three cities (Shenyang, Anshan and Jinzhou) in China. A total of 28830 people were selected; the overall response rate was $87.4 \%$ (25 196/28830). The overall prevalence of hypertension was $28.7 \%(7237 / 25196)$. Of those with hypertension, $42.9 \%$ (3107/7237) were aware of their condition, $28.2 \%(2042 / 7237)$ were receiving treatment and 3.7\% (264/7237) were considered controlled. More than half (57.8\%) of the hypertensives did not think that high BP would endanger their lives. Multivariate analysis revealed that age, gender, education, occupation, income, body mass index, waist circumference and a family history of hypertension correlated significantly with the prevalence of hypertension. Among all the hypertensives, higher awareness (determined by odds ratios; $95 \%$ confidence intervals) was noted for persons who were white-collar workers $(1.29 ; 1.08,1.53$, respectively), overweight $(1.48 ; 1.30,1.69)$, obese $(3.37 ; 2.76,4.11)$ or had a family history of hypertension $(3.07 ; 2.76,3.42)$. Among the individuals aware of their hypertension, treatment was more common in those with a higher level of education and less common among individuals consuming $\geqslant 2$ alcoholic drinks per day $(0.65 ; 0.52,0.83)$. Controlled hypertension was much less common among older persons, and participants who were former smokers $(0.49 ; 0.26,0.91)$. The results indicate that more attention is needed to improve the awareness of the potentially fatal nature of hypertension in urban China.
\end{abstract}

Hypertension Research (2011) 34, 1087-1092; doi:10.1038/hr.2011.99; published online 21 July 2011

Keywords: awareness; control; hypertension prevalence; risk factors; treatment

\section{INTRODUCTION}

In the past decade, China has experienced rapid economic development, resulting in both lifestyle and dietary changes. Cardiovascular disease (CVD) has since become the leading cause of death in China, ${ }^{1}$ and hypertension has been identified as the major risk factor for CVD and other vascular diseases. ${ }^{2,3}$ It has been shown that promoting the awareness of established risk factors and early management is an essential starting point in the prevention of increased burden of morbidity and mortality due to hypertension-related CVDs. ${ }^{4}$ However, in China, especially in urban China, information on factors associated with awareness, treatment and control of hypertension is scarce, despite the increasing prevalence of hypertension. In a systematic Medline search, we identified five surveys conducted in the most recent 10 -year period concerning the prevalence, awareness, treatment and control of hypertension among the general population in China. Only two studies focused on urban populations; one conducted among urban-dwelling Chinese individuals aged $\geqslant 45$ years, ${ }^{5}$ and another conducted among urban-dwelling individuals aged $\geqslant 60$ years. $^{6}$ To explore the prevalence, awareness, treatment and control of hypertension in an urban Chinese population, 25196 subjects from 33 communities in three cities of northeast China were surveyed between 2009 and 2010 .

\section{METHODS}

Sample design

The subjects were selected for the CHPSNE Study (Control Hypertension and Other Risk Factors to Prevent Stroke with Nutrition Education in Urban Area of northeast China). The CHPSNE Study used a three-stage stratified sampling

${ }^{1}$ Division of Neurology, The Fourth Affiliated Hospital of China Medical University, Shenyang, PR China and ${ }^{2}$ Department of Biostatistics and Epidemiology, School of Public Health, China Medical University, Shenyang, PR China

Correspondence: Professor G-H Dong, Department of Biostatistics and Epidemiology, School of Public Health, China Medical University, 92 North 2nd Road, Heping District, Shenyang 110001, PR China.

E-mail: ghdong@mail.cmu.edu.cn

Received 1 December 2010; revised 11 March 2011; accepted 7 April 2011; published online 21 July 2011 


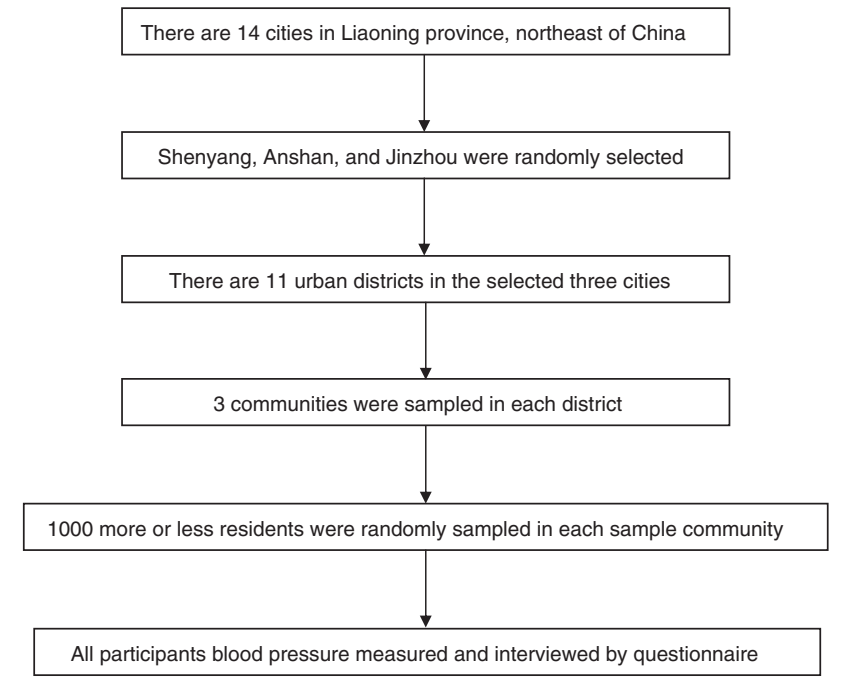

Figure 1 Sampling procedure of the CHPSNE study (Control Hypertension and Other Risk Factors to Prevent Stroke with Nutrition Education in Urban Area of northeast China).

method to select a sample representative of the general urban population aged 18-74 years (see Figure 1). More than 20 million people reside in 14 cities in the Liaoning province in northeast China. These 14 cities were stratified into three socioeconomic brackets: low, moderate and high. In April 2009, three cities (Shenyang, Anshan and Jinzhou) were randomly selected from these three zones. There are five urban districts in Shenyang, three urban districts in Anshan and three urban districts in Jinzhou. Three communities were randomly selected from each urban district of the selected cities, resulting in a total of 33 communities included in the study. The final stage of sampling was stratification by gender ( $50 \%$ men and $50 \%$ women) and by age distribution based on the 1990 Chinese census data. Only one participant (with $\geqslant 5$ years of residency) was selected from each household, without replacement. This study was approved by the ethics committee of China Medical University. All subjects were included only after they had signed an informed-consent form. Subjects were excluded from the survey if they were not willing to complete all required questions.

A total of 28830 subjects were randomly selected from 33 communities and were invited to participate in the study. A total of 25196 people (12413 men and 12783 women) completed the survey and examination. The overall response rate was $87.4 \%$ ( $86.1 \%$ in men and $88.7 \%$ in women). The age of the subjects surveyed and examined ranged from 18 to 74 years of age, with an average age of $41.7 \pm 14.4$ years.

\section{Blood pressure measurement}

Three blood pressure (BP) measurements were obtained from each participant by trained and certified medical personnel according to a standard protocol recommended by the American Heart Association. ${ }^{7}$ BP was measured three times after $5 \mathrm{~min}$ of rest with each participant in the seated position, while allowing the patient a 2-min resting interval between the three BP measurements. In addition, participants were advised to avoid alcohol, cigarette smoking, coffee/tea and exercise for at least $30 \mathrm{~min}$ before their BP measurement. A standardized mercuric-column sphygmomanometer was used, and the most appropriate of the four cuff sizes (pediatric, regular adult, large or thigh) was chosen on the basis of the circumference of the participant's arm.

All study investigators and staff members successfully completed a training program that oriented them both to the aims of the study and to the specific tools and methodologies to be employed. At the training sessions, interviewers were given detailed instructions on administration of the study questionnaire. All BP medical personnel participated in a special training session on the use of a standardized protocol for the measurement of BP. To be certified as a BP observer, individuals were required to demonstrate satisfactory performance on a written examination, which tested knowledge of preparing study participants for the procedure of measuring $\mathrm{BP}$, selecting the correct cuff size and using the standard BP measurement techniques. In addition, medical personnel completed a videotaped protocol of the technique for BP measurement during a standardized examination, and concordant measurements with an instructor were required.

\section{Diagnostic criteria}

The hypertension status of the subjects was assessed based on the US Seventh Joint National Committee report on the prevention, detection, evaluation and treatment of high BP. ${ }^{8}$ Hypertension was defined as an average systolic BP $\geqslant 140 \mathrm{~mm} \mathrm{Hg}$ or an average diastolic $\mathrm{BP} \geqslant 90 \mathrm{~mm} \mathrm{Hg}$, or self-reported current treatment for hypertension with an antihypertensive medication within the 2 weeks before the interview. This definition excludes patients previously qualified as hypertensive who have been reduced to a non-hypertensive range solely by the use of non-pharmacologic measures. The awareness of hypertension was defined by any self-report of hypertension due to any prior diagnosis of hypertension by a health-care professional among the population defined as having hypertension. The treatment of hypertension was defined as the selfreported use of pharmacological medication for the management of high BP within the 2 weeks preceding the participant's interview. Control of hypertension was defined as pharmacological treatment of hypertension associated with an average systolic BP $<140 \mathrm{~mm} \mathrm{Hg}$ and an average diastolic BP $<90 \mathrm{~mm} \mathrm{Hg}$.

During the clinic visit, height, weight and waist circumference were measured using a standardized protocol and body mass index, which was calculated in $\mathrm{kg} \mathrm{m}^{-2}$. Normal weight, overweight and obesity were defined as having a calculated body mass index of $<25,25-30$ and $\geqslant 30 \mathrm{~kg} \mathrm{~m}^{-2}$, respectively. Information on demographics (that is age, gender and residential area), socioeconomic status (that is education level achieved, occupation and annual household income), cigarette smoking, alcohol consumption, a family history of hypertension, medical treatment of conditions related to hypertension and other information was collected with a standardized questionnaire that has been used for other studies in China. ${ }^{9} 10$ Participants who reported having smoked $\geqslant 100$ cigarettes during their lifetime were classified as current smokers if they currently smoked and former smokers if they did not. The questionnaire section regarding consumption of alcohol included the number of liangs $(\approx 50 \mathrm{~g})$ of rice wine, wine, beer or liquor consumed during the preceding 12 months. Heavy alcohol consumption was categorized as $>2$ drinks per day, with one drink equivalent to $355 \mathrm{~g}$ of beer, $100 \mathrm{~g}$ of rice wine or wine, or $25 \mathrm{~g}$ of liquor. Finally, information was collected regarding the time lapse between the patient's diagnosis of hypertension by a health-care professional and their follow-up BP measurement. The periods were categorized as being within 3 months, 3-5 months, $>5$ months or never. We also asked questions to collect information about (1) people's perception of high BP and life endangerment and recognition of the seriousness of hypertension and (2) the reasons for non-compliance when utilizing therapeutic interventions. The actual questions asked were the following. (1) Do you think that high BP can endanger your life (yes or no)? (2) Why did you not take the prescribed medication after becoming aware of your hypertension diagnosis (unaffordable drug prices; a deficiency in recognition of the seriousness of hypertension; a lack of guidance for proper utilization of medication; often forgetting to take medication because of a busy work life; other reasons)?

\section{Statistical methods}

The population was stratified by age into six groups: aged 18-24, 25-34, 35-44, 45-54, 55-64 and 65-74 years. Continuous variables were given as the mean \pm s.d., but categorical variables were presented as the percentage in each subgroup. The associations between categorical variables were tested by the use of contingency tables and the $\chi^{2}$ test. The comparisons between continuous variables between groups were performed using the Student's $t$-test. We calculated adjusted odds ratios (ORs) with 95\% confidence intervals (CIs) for hypertension status with multivariate logistic regression models. All data analyses were conducted using SAS software (Version 9.1; SAS Institute, Cary, NC, USA). All statistical tests were two-tailed, and $P$-values $<0.05$ were considered statistically significant. 


\section{RESULTS}

Table 1 shows the characteristics of the subjects. Overall, $28.7 \%$ $(n=7237)$ of the Chinese urban adult population aged 18-74 years had hypertension. Among adults 35-74 years old, the prevalence of hypertension was $39.1 \%$. Only $42.9 \%$ (3107/7237) of those with hypertension were aware of their diagnosis. Of all the hypertensive subjects in the study population, 28.2\% (2042/7237) were taking a prescribed medication to lower their BP, and only 3.7\% (264/7237) had controlled hypertension. Of those aware of their hypertension diagnosis, 65.7\% (2042/3107) were treated, and 12.9\% (264/2042) of those treated became normotensive with treatment by their study visit.

Hypertension prevalence and awareness were more common among participants $\geqslant 55$ years old (Table 2 ). Additionally, participants who were female, white-collar workers, overweight or obese, former smokers and those with a family history of hypertension were more likely to be aware of their diagnosis of hypertension. In contrast, subjects whose highest level of education was primary school, who

Table 1 Characteristics of the subjects $(N=25196)$

\begin{tabular}{|c|c|c|}
\hline Variables & $\mathrm{n}$ & $\%$ \\
\hline \multicolumn{3}{|l|}{ Age groups (years) } \\
\hline $18-34$ & 9820 & 39.0 \\
\hline $35-44$ & 5253 & 20.9 \\
\hline $45-54$ & 5158 & 20.5 \\
\hline $55-64$ & 3096 & 12.3 \\
\hline $65-74$ & 1869 & 7.4 \\
\hline Gender: female & 12783 & 50.7 \\
\hline \multicolumn{3}{|l|}{ Educational level } \\
\hline No school & 983 & 3.9 \\
\hline Primary school & 3225 & 12.8 \\
\hline Junior high school & 14736 & 58.5 \\
\hline$\geqslant$ Senior high school & 6252 & 24.8 \\
\hline \multicolumn{3}{|l|}{ Career } \\
\hline Blue-collar worker & 11887 & 47.2 \\
\hline White-collar worker & 4011 & 15.9 \\
\hline Organization personnel cadres & 1894 & 7.5 \\
\hline Others & 7404 & 29.4 \\
\hline \multicolumn{3}{|l|}{ Body weight } \\
\hline Normal & 16567 & 65.8 \\
\hline Overweight & 7318 & 29.0 \\
\hline Obesity & 1311 & 5.2 \\
\hline$W C(\mathrm{~cm}):$ male $\geqslant 85$ or female $\geqslant 80$ & 11517 & 45.7 \\
\hline Family history of hypertension & 8313 & 33.0 \\
\hline \multicolumn{3}{|l|}{ Cigarette smoking } \\
\hline Never & 17180 & 68.2 \\
\hline Current & 7094 & 28.2 \\
\hline Former & 922 & 3.7 \\
\hline Alcohol consumption $\geqslant 2$ drinks per day & 5114 & 20.3 \\
\hline Like eating fried food: yes & 7831 & 31.1 \\
\hline Control diet with low calorie and fat: yes & 5376 & 21.3 \\
\hline Prevalence of hypertension & 7237 & 28.7 \\
\hline Awareness among hypertensives & 3107 & 42.9 \\
\hline Treatment among hypertensives & 2042 & 28.2 \\
\hline Controlled among hypertensives & 264 & 3.7 \\
\hline
\end{tabular}

Abbreviation: WC, waist circumference.
Table 2 Percentage (\%) of prevalence, awareness, treatment and control of hypertension by socio-demographics and those with and without risk factors

\begin{tabular}{|c|c|c|c|c|}
\hline Variables & $\begin{array}{c}\text { Hypertension } \\
(\mathrm{n}=7237)^{\mathrm{a}}\end{array}$ & $\begin{array}{l}\text { Awareness } \\
(\mathrm{n}=3107)^{\mathrm{b}}\end{array}$ & $\begin{array}{c}\text { Treatment } \\
(\mathrm{n}=2042)^{\mathrm{c}}\end{array}$ & $\begin{array}{c}\text { Control } \\
(\mathrm{n}=264)^{\mathrm{d}}\end{array}$ \\
\hline \multicolumn{5}{|l|}{ Age groups (years) } \\
\hline $18-34$ & 12.4 & 24.3 & 31.1 & 47.8 \\
\hline $35-44$ & $23.7^{* *}$ & $39.5^{* *}$ & $56.8^{* *}$ & $8.6^{* *}$ \\
\hline $45-54$ & $38.7^{* *}$ & $45.3^{* *}$ & $68.8^{* *}$ & $13.8^{* *}$ \\
\hline $55-64$ & $54.5^{* *}$ & $54.2^{* *}$ & $73.5^{* *}$ & $10.9 * *$ \\
\hline $65-74$ & $58.4^{* *}$ & $46.0^{* *}$ & $75.1^{* *}$ & $9.8^{* *}$ \\
\hline \multicolumn{5}{|l|}{ Gender } \\
\hline Female & 24.7 & 51.5 & 71.7 & 13.5 \\
\hline Male & $32.9 * *$ & $36.3^{* *}$ & 59.1 ** & 12.2 \\
\hline \multicolumn{5}{|l|}{ Educational level } \\
\hline No school & 45.4 & 37.9 & 61.5 & 7.7 \\
\hline Primary school & $38.1^{* *}$ & $49.8^{* *}$ & $77.6^{* *}$ & 12.2 \\
\hline Junior high school & $29.3^{* *}$ & $43.2^{*}$ & 63.9 & $15.4^{*}$ \\
\hline$\geqslant$ Senior high school & $19.8^{* *}$ & 37.1 & 59.0 & 5.2 \\
\hline \multicolumn{5}{|l|}{ Career } \\
\hline Blue-collar worker & 26.1 & 42.3 & 69.1 & 12.3 \\
\hline White-collar worker & 27.7 & $46.4^{*}$ & 73.6 & 13.5 \\
\hline Organization personnel cadres & $36.2^{* *}$ & 41.0 & 65.1 & 8.2 \\
\hline Others & $31.7^{* *}$ & 42.7 & $57.4^{* *}$ & 15.1 \\
\hline \multicolumn{5}{|l|}{ Family income per year } \\
\hline Quartile 1 (lowest) & 38.5 & 40.8 & 67.2 & 9.5 \\
\hline Quartile 2 & $30.3^{* *}$ & $54.5^{* *}$ & 69.6 & 14.3 \\
\hline Quartile 3 & $28.0 * *$ & 41.3 & 68.6 & 12.0 \\
\hline Quartile 4 (highest) & $25.7^{* *}$ & 40.7 & $55.2^{* *}$ & $16.5^{*}$ \\
\hline \multicolumn{5}{|l|}{ Body weight } \\
\hline Normal & 20.1 & 32.8 & 68.5 & 9.6 \\
\hline Overweight & $42.0 * *$ & $49.0 * *$ & 66.9 & $12.6^{*}$ \\
\hline Obesity & $62.9 * *$ & $61.4^{* *}$ & $56.3^{* *}$ & $22.8^{* *}$ \\
\hline \multicolumn{5}{|l|}{$W C(\mathrm{~cm})$} \\
\hline Male $<85$ or female $<80$ & 17.2 & 28.8 & 65.0 & 11.6 \\
\hline Male $\geqslant 85$ or female $\geqslant 80$ & $42.4^{* *}$ & $49.7^{* *}$ & 65.9 & 13.3 \\
\hline \multicolumn{5}{|l|}{ Family history of hypertension } \\
\hline No & 23.6 & 31.6 & 64.3 & 11.7 \\
\hline Yes & $39.1^{* *}$ & $56.8^{* *}$ & 66.7 & 13.7 \\
\hline \multicolumn{5}{|l|}{ Cigarette smoking } \\
\hline Never & 27.1 & 46.1 & 67.9 & 12.8 \\
\hline Current & $30.6^{* *}$ & $35.7^{* *}$ & $59.7^{* *}$ & 12.5 \\
\hline Former & $43.8^{* *}$ & 45.5 & 66.3 & 15.6 \\
\hline \multicolumn{5}{|l|}{ Alcohol consumption } \\
\hline$<2$ drinks per day & 27.7 & 45.7 & 69.4 & 13.7 \\
\hline$\geqslant 2$ drinks per day & $32.8^{* *}$ & $33.8^{* *}$ & $49.2^{* *}$ & $8.2^{*}$ \\
\hline \multicolumn{5}{|l|}{ Like eating fried food } \\
\hline Never & 30.2 & 44.0 & 67.5 & 12.9 \\
\hline Yes & $23.3^{* *}$ & $37.8^{* *}$ & $55.9 * *$ & 12.8 \\
\hline \multicolumn{5}{|c|}{ Control diet with low calorie and fat } \\
\hline Never & 27.2 & 41.8 & 61.1 & 13.3 \\
\hline Yes & $32.0 * *$ & $45.0 * *$ & $73.9 * *$ & 12.5 \\
\hline
\end{tabular}

Among all subjects.

Among subjects with hypertension.

cAmong subjects aware of their hypertension diagnosis.

${ }^{\mathrm{d}}$ Among subjects receiving pharmacological treatment because of their hypertension. ${ }^{*} P<0.05 ;{ }^{* *} P<0.01$ 
Table 3 Conditions about blood pressure in hypertensives aware of having hypertension in urban adult population, aged 18-74 years, in China, 2009-2010

\begin{tabular}{lcc}
\hline Condition of hypertension & Male & Female \\
\hline Periodicity of BP measurement & & \\
$\quad<1$ week & $499(33.7)$ & $626(38.5)$ \\
$<1$ month & $374(25.3)$ & $430(26.4)$ \\
$<3$ months & $221(14.9)$ & $290(17.9)$ \\
$<6$ months & $176(11.9)$ & $150(9.2)$ \\
$>6$ months & $58(3.9)$ & $46(2.8)$ \\
Never measuring BP & $152(10.3)$ & $85(5.2)$ \\
& & \\
Conditions of taking antihypertensive medication per year & \\
Regularity & $395(26.7)$ & $465(28.6)$ \\
Interruption & $331(22.3)$ & $517(31.7)$ \\
Occasionally & $149(10.1)$ & $185(11.4)$ \\
Never taking medicine & $605(40.9)$ & $460(28.3)$ \\
& & \\
Effect of antihypertensive medication ${ }^{\mathrm{b}}$ & & \\
Good & $88(10.1)$ & $150(12.8)$ \\
Acceptable & $271(31.0)$ & $366(31.4)$ \\
Dys-effect & $438(50.1)$ & $569(48.8)$ \\
Never measuring BP & $78(8.9)$ & $82(7.0)$ \\
& & \\
The cause of not taking antihypertensive medication & \\
Lack of knowledge about dangerous & $155(25.6)$ & $136(29.6)$ \\
of hypertension & & \\
Financial straits & $198(32.7)$ & $173(37.6)$ \\
Lack guidance & $87(14.4)$ & $39(8.5)$ \\
Troublesome & $34(5.6)$ & $57(12.4)$ \\
Other & $131(21.7)$ & \\
\hline
\end{tabular}

Abbreviation: BP, blood pressure.

Data are $n(\%)$.

aThe time of taking antihypertensive medication per year, regularity: $>9$ months per year;
interruption: 3 months per year to 9 months per year; occasionally: $<3$ months per year.

bThe time of controlling the BP (systolic BP $<140 \mathrm{~mm} \mathrm{Hg}$ and diastolic BP $<90 \mathrm{~mm} \mathrm{Hg}$ ) per year, good: $>9$ months; acceptable: $>6$ months; dys-effect: $<6$ months.

were current smokers and who consumed $\geqslant 2$ servings of alcohol per day were less likely to be aware of their hypertension. Among those who were aware of their hypertension, participants who were older, women and whose diets consisted of low-calorie and low-fat foods were more likely to be receiving medications for their hypertension. Individuals who had a higher income, who were obese, who were current smokers, who consumed $\geqslant 2$ alcoholic drinks per day and who consumed additional fried food were less likely to be receiving medications for their hypertension. Among participants receiving treatment for their hypertension, achieving BP control was more common in those individuals with the highest income and in individuals who were overweight or obese. BP control in former smokers $(15.6 \%)$ was higher than BP control achieved in current smokers $(12.5 \%)$ but lacked a statistically significant difference $(P>0.05)$.

As shown in Table 3, of the hypertensives aware of having hypertension, only $36.2 \%$ reported that they were measuring their BP at least once per week, and only $27.7 \%$ took antihypertensive medication for their hypertension regularly. Of all the treated patients, only $10.1 \%$ of men and $12.8 \%$ of women maintained normotensive BP for at least 9 months out of the year. The most prominent reasons given by hypertensive patients aware of having hypertension but not taking their antihypertensive medication were a lack of money $(34.8 \%)$ and a lack of knowledge about the potential mortality associated with uncontrolled hypertension (27.3\%). Of the studied hypertensive patients, $57.8 \%$ (4183/7237) did not think that high BP would endanger their lives. This potentially fatal misunderstanding occurred at an even higher rate in those unaware of their hypertension $(68.1 \%)$ compared with individuals who were aware of their hypertension diagnosis (38.0\%). Among those treatment non-compliant patients who were aware of their hypertension diagnosis but not taking their prescribed medication to lower their BP, only $48.9 \%$ of patients recognized the fatalities associated with hypertension, and $34.8 \%(371 / 1065)$ cited unaffordable drug prices as the reason for non-compliance.

After multiple logistic regression analysis, age, gender, smoking status, alcohol consumption, education level, occupation, body mass index, waist circumference and a family history of hypertension were shown as the most pertinent risk factors for an individual to be diagnosed with hypertension (Table 4). A higher level of education was shown to be associated with qualities protective against the development of hypertension. Family income level was inversely related to the prevalence of hypertension; that is, higher-income families had a lower prevalence of hypertension. Study subjects who had worked in an organization cadre appeared to have a 1.37 -fold higher risk for developing hypertension than blue-collar workers (95\% CI: 1.20-1.55). Compared with subjects of normal weight, overweight subjects or obese subjects were more likely to be hypertensive (OR: 1.91 and 5.45, respectively). Among those aware of having hypertension, participants who were at least 35 years old, who were female, who had a higher level of education, who were white-collar workers, who were overweight or obese, who had a family history of hypertension and who controlled their diet with low-calorie and low-fat foods were more likely to be receiving pharmacological treatment for their hypertension. In contrast, those subjects consuming $\geqslant 2$ alcoholic drinks per day were significantly less likely to be receiving antihypertensive medication (OR: 0.84; 95\% CI: 0.73-0.98). Among individuals receiving treatment for their hypertension, the OR $(95 \% \mathrm{CI})$ of the hypertension control group composed of white-collar workers was $1.92(1.23,2.00)$ and was $2.27(1.40-3.71)$ for those individuals considered obese.

\section{DISCUSSION}

The results of this study constitute the most reliable and current information on the current prevalence of hypertension, the extent to which hypertension is being treated and controlled and the risk factors for the disease among the urban adult population in northeast China. This study was conducted on a large representative sample of adult Chinese individuals living in an urban setting using standard protocols and instruments. Strict training programs for data collectors and thorough quality assurance programs were used to ensure the quality of the data collection. Additional strengths of the study include a high response rate, the use of three separate $\mathrm{BP}$ measurements, collection of detailed information on each individual's history and family history of hypertension and obtaining precise information on pharmaceutical treatment utilization. For all of these reasons, these data likely constitute the most accurate estimates of hypertension detection, treatment, control and data pertaining to the risk factors correlated with the development and time course of the disease that are available for the general urban Chinese population living in northeast China.

Overall, the prevalence of hypertension identified in the present study exceeds that of many developing counties ${ }^{11,12}$ and is similar to, or higher than, that observed in developed counties. ${ }^{13-15}$ Moreover, the proportion of individuals with hypertension aware, treated and controlled was unacceptably low in urban Chinese. The results also 
Table 4 Prevalence $(\%)$ and adjusted OR $(95 \% \mathrm{Cl})$ of factors associated with hypertension, awareness, treatment and control in urban Chinese from 33 communities, China ${ }^{\mathrm{a}}$

\begin{tabular}{|c|c|c|c|c|}
\hline Variables & $\begin{array}{l}\text { Hypertension OR } \\
(95 \% \mathrm{Cl})^{\mathrm{b}}\end{array}$ & $\begin{array}{c}\text { Awareness OR } \\
(95 \% \mathrm{Cl})^{\mathrm{C}}\end{array}$ & $\begin{array}{l}\text { Treatment OR } \\
\qquad(95 \% \mathrm{Cl})^{\mathrm{d}}\end{array}$ & $\begin{array}{l}\text { Control OR } \\
(95 \% \mathrm{Cl})^{\mathrm{e}}\end{array}$ \\
\hline \multicolumn{5}{|l|}{ Age groups (ref: 18-34 years old) } \\
\hline 35-44 & $2.22(2.01-2.45)$ & $2.28(1.87-2.77)$ & $2.50(1.77-3.53)$ & $0.02(0.01-0.06)$ \\
\hline $45-54$ & $4.54(4.11-5.01)$ & $3.01(2.49-3.65)$ & $3.93(2.78-5.55)$ & $0.03(0.01-0.07)$ \\
\hline 55-64 & $9.54(8.51-10.70)$ & $4.89(3.99-6.00)$ & $4.22(2.95-6.04)$ & $0.02(0.01-0.05)$ \\
\hline 65-74 & $14.45(12.56-16.63)$ & $4.91(3.90-6.19)$ & $4.80(3.21-7.20)$ & $0.02(0.01-0.040$ \\
\hline Gender (ref: female) & $2.07(1.91-2.25)$ & $0.79(0.69-0.90)$ & $0.90(0.72-1.12)$ & $0.73(0.48-1.09)$ \\
\hline \multicolumn{5}{|l|}{ Educational level (ref: no school) } \\
\hline Primary school & $0.81(0.69-0.96)$ & $1.41(1.10-1.79)$ & $2.57(1.76-3.76)$ & $1.88(0.84-4.19)$ \\
\hline Junior high school & $0.83(0.71-0.97)$ & $1.51(1.19-1.92)$ & $1.91(1.32-2.75)$ & $1.89(0.85-4.19)$ \\
\hline$\geqslant$ Senior high school & $0.81(0.67-0.97)$ & $1.70(1.27-2.26)$ & $1.91(1.21-3.01)$ & $0.12(0.04-0.39)$ \\
\hline \multicolumn{5}{|l|}{ Occupation (ref: blue-collar worker) } \\
\hline White-collar worker & $0.92(0.83-1.03)$ & $1.29(1.08-1.53)$ & $1.47(1.11-1.95)$ & $1.92(1.23-2.99)$ \\
\hline Organization personnel cadres & $1.37(1.20-1.55)$ & $1.23(1.01-1.50)$ & $0.97(0.70-1.33)$ & $1.10(0.58-2.07)$ \\
\hline Others & $1.27(1.17-1.37)$ & $1.25(1.10-1.42)$ & $0.64(0.53-0.78)$ & $1.23(0.87-1.75)$ \\
\hline \multicolumn{5}{|l|}{ Family income per year (ref: Quartile 1) } \\
\hline Quartile 2 & $0.82(0.72-0.93)$ & $1.63(1.34-1.98)$ & $1.16(0.87-1.56)$ & $1.51(0.89-2.56)$ \\
\hline Quartile 3 & $0.94(0.85-1.04)$ & $1.01(0.86-1.19)$ & $1.37(1.06-1.78)$ & $0.95(0.58-1.56)$ \\
\hline Quartile 4 (highest) & $1.20(1.06-1.35)$ & $1.05(0.87-1.28)$ & $0.95(0.70-1.29)$ & $1.56(0.89-2.73)$ \\
\hline \multicolumn{5}{|l|}{ Body weight (ref: normal) } \\
\hline Overweight & $1.91(1.76-2.07)$ & $1.48(1.30-1.69)$ & $1.02(0.83-1.27)$ & $1.42(0.96-2.11)$ \\
\hline Obesity & $5.45(4.73-6.29)$ & $3.37(2.76-4.11)$ & $1.03(0.77-1.38)$ & $2.27(1.40-3.71)$ \\
\hline WC $(\mathrm{cm})$ (ref: male $<85$ or female $<80$ ) & $1.66(1.54-1.80)$ & $1.64(1.43-1.88)$ & $1.22(0.97-1.54)$ & $0.60(0.39-0.92)$ \\
\hline Family history of hypertension (ref: no) & $2.47(2.31-2.64)$ & $3.07(2.76-3.42)$ & $1.19(1.01-1.42)$ & $0.97(0.71-1.32)$ \\
\hline \multicolumn{5}{|l|}{ Cigarette smoking (ref: never) } \\
\hline Current & $1.28(1.17-1.39)$ & $1.09(0.95-1.25)$ & $0.95(0.77-1.19)$ & $0.97(0.62-1.51)$ \\
\hline Former & $1.04(0.88-1.22)$ & $0.96(0.76-1.22)$ & $0.77(0.53-1.12)$ & $0.49(0.26-0.91)$ \\
\hline Alcohol consumption (ref: $<2$ drinks per day) & $1.24(1.14-1.36)$ & $0.84(0.73-0.98)$ & $0.65(0.52-0.83)$ & $0.21(0.11-0.40)$ \\
\hline Like eating fried food (ref: never) & $0.82(0.75-0.89)$ & $1.04(0.90-1.20)$ & $0.73(0.58-0.91)$ & $1.02(0.63-1.66)$ \\
\hline Control diet with low calorie and fat (ref: never) & $1.03(0.96-1.11)$ & $1.12(1.00-1.26)$ & $1.58(1.32-1.88)$ & $1.22(0.91-1.65)$ \\
\hline
\end{tabular}

Abbreviations: $\mathrm{Cl}$, confidence interval; OR, odds ratio; WC, waist circumference.

${ }^{a}$ Adjusted for age, sex, education level, occupation, family income, overweight, obesity, former and current smoking, alcohol consumption, family history of hypertension and behavior of bite and

sup.
bAmong all subjects.

CAmong subjects with hypertension.

${ }^{d}$ Among subjects aware of their hypertension diagnosis.

eAmong subjects receiving pharmacological treatment because of their hypertension.

showed that most hypertensive patients, whether unaware or aware of their hypertension diagnosis, did not think that high BP would endanger their lives. Furthermore, among those non-compliant hypertensive patients who were not taking prescribed medication to lower their BP, only $48.9 \%$ recognized the fatalness of hypertension. Similar to other economically developing countries, the health-care system in China faces financial challenges. Although national guidelines require $\mathrm{BP}$ to be measured during all outpatient visits, it is still very common that a BP check-up is not performed in urban China during outpatient visits. In addition, community-based BP screening and education programs are still uncommon in urban areas of China. Our findings highlight the need for developing a national high BP education program to coordinate the efforts of detection, prevention and treatment of hypertension in China.

Studies from several countries have investigated the factors associated with the prevalence, awareness, treatment and ultimate control of hypertension. ${ }^{5,6,11,14,15}$ Our study findings are consistent with several of the previous reports. Compared with patients who continue to smoke, patients who quit smoking may be more health conscious and visit physicians more often after a diagnosis of hypertension. Physicians may pay more attention to patients with hypertension who are overweight or obese because they are at a greater risk for developing CVDs. Studies conducted in developing countries have consistently indicated that, compared to the people with a lower average income, people with a higher income have better access to health care and better awareness, treatment and control of their hypertension. However, in contrast to these previous studies, our investigation revealed that among those individuals aware of their hypertension, treatment was less common among those individuals having the highest level of income (OR: 0.95; 95\% CI: 0.70-1.29). Among urban Chinese subjects with a higher income, $32.3 \%$ cited often forgetting to take medication because of a busy work schedule as the reason for non-compliance. This reason for non-compliance was higher when compared with their lower-income counterparts 
(11.5\%). These results may suggest an association of individuals with higher incomes with lower levels of treatment among the urban Chinese. The lower levels of treatment may be explained by higherincome subjects having such a rigorous work schedule that they forget to take their medication.

Our study presents data that may suggest an association between hypertension and a number of risk factor differences between organization cadres and blue-collar workers. Working in organization cadres appears to be associated with a higher risk of developing hypertension than blue-collar employment. Compared with bluecollar workers, organization personnel cadres have a relatively higher education, a more regular work schedule and spend more time sitting in front of their computers. Accordingly, individuals employed in organization personnel cadres may not get enough exercise and/or may suffer from mental health problems due to work-related stress. In addition, in the present study, $62.7 \%$ of organization cadre personnel were male, and the average age of organization cadre personnel ( $44.5 \pm 16.3$ years) was higher than that of blue-collar workers (41.2 \pm 14.4 years). The associations between education level and hypertension awareness, treatment and control have remained inconsistent. In the SUNSET study, ${ }^{14}$ there were no associations between education level and hypertension awareness, treatment and control groups of individuals of Indian and African ethnicity, but in the white Dutch hypertensives. In a multiethnic Asian population's study, lower education level was associated with poor BP control. ${ }^{15} \mathrm{On}$ the contrary, consistent with the results of Scottish MONICA surveys, ${ }^{16}$ we found lower control rates among those with higher education levels, which indicated that education is a strong socioeconomic predictor of awareness and treatment, but not of control. A family history of hypertension is a well-established risk factor for hypertension. ${ }^{17}$ However, very little is known about the associations between a family history of hypertension and the awareness, treatment and control of hypertension. One study that assessed a family history of hypertension and BP control found no association. ${ }^{18}$ However, in the SUNSET study, ${ }^{14}$ a family history of hypertension was a strong predictor of hypertension awareness and subsequent treatment in the Dutch ethnic groups and was associated with better BP control in subjects of African ethnicity. In the present study, however, we found that a family history of hypertension was associated only with awareness and treatment of hypertension. These findings may imply that the presence of a family history of hypertension may positively influence individuals to check their BP and to seek medical intervention. Alternatively, physicians may pay more attention to patients with a family history of hypertension because they are at a greater risk of CVD.

Findings in the current investigation must be considered within the context of the study's limitations. Specifically, BP was measured three times following a standardized protocol during only a single visit. According to both the World Health Organization and the National Institute of Health guidelines, hypertension should be defined based on the average of at least two or more BP readings taken at two or more visits after an initial screening. ${ }^{19,20}$ Another limitation of the current study was the cross-sectional study design and that the maximum age of the surveyed participants was only 74 years old.

In conclusion, the findings of this study have important public health implications. First, more attention should be directed to improving cognizance of the fatalness of hypertension in urban adults of China. Second, more effort is needed to promote hypertension awareness, treatment and control among those individuals not only with higher hypertension risk but also among those with lower hypertension risk. Finally, control of hypertension was lower among current smokers and among persons who consume $\geqslant 2$ alcoholic drinks per day. Smoking cessation and reduced alcohol consumption may not be the sole reason for controlled hypertension among these patients, but in these individuals, these changes may confer additional benefits along with other concurrent lifestyle modifications.

\section{CONFLICT OF INTEREST}

The authors declare no conflict of interest.

\section{ACKNOWLEDGEMENTS}

We are very grateful to the participants from northeast China. We also thank the anonymous reviewers for their very helpful comments. This research was supported by grants from the Liaoning Province Education Department Foundation (L2010672).

1 He J, Gu D, Wu X, Reynolds K, Duan X, Yao C, Wang J, Chen CS, Chen J, Wildman RP, Klag MJ, Whelton PK. Major causes of death among men and women in China. N Engl J Med 2005; 353: 1124-1134.

2 Lawes CM, Vander HS, Rodgers A. Global burden of blood-pressure-related disease, 2001. Lancet 2008; 371: 1513-1518.

3 Lee DS, Massaro JM, Wang TJ, Kannel WB, Benjamin EJ, Kenchaiah S, Levy D, D'Agostino Sr RB, Vasan RS. Antecedent blood pressure, body mass index, and the risk of incident heart failure in later life. Hypertension 2007; 50: 869-876.

4 Barengo NC, Kastarinen M, Antikainen R, Nissinen A, Tuomilehto J. The effects of awareness, treatment and control of hypertension on cardiovascular and all-cause mortality in a community-based population. J Hum Hypertens 2009; 23: 808-816.

5 Xu L, Wang S, Wang YX, Wang YS, Jonas JB. Prevalence of arterial hypertension in the adult population in rural and urban China: the Beijing eye study. Am J Hypertens 2008; 21: 1117-1123.

6 Zhang X, Zhu M, Dib HH, Hu J, Tang S, Zhong T, Ming X. Knowledge, awareness, behavior $(\mathrm{KAB})$ and control of hypertension among urban elderly in western China. Int J Cardiol 2009; 137: 9-15.

7 Crundy SM, Brewer Jr HB, Cleeman JI, Smith Jr SC, Lenfant C. Definition of metabolic syndrome: report of the National Heart, Lung, and Blood Institute/American heard Association conference on scientific issues related to definition. Circulation 2004; 109: 433-438.

8 Chobanian AV, Bakris GL, Black HR, Cushman WC, Green LA, Izzo Jr JL, Jones DW, Materson BJ, Oparil S, Wright Jr JT, Roccella EJ. Seventh report of the Joint National Committee on Prevention, Detection, Evaluation, and Treatment on High Blood Pressure. Hypertension 2003; 42: 1206-1252.

9 Dong G, Sun Z, Zheng L, Li J, Zhang X, Zhang X, Xu C, Hu D, Sun Y. Prevalence, awareness, treatment, and control of hypertension in rural adults from Liaoning Province, northeast China. Hypertens Res 2007; 30: 951-958.

10 Wang Z, Wu Y, Zhao L, Li Y, Yang J, Zhou B. Trends in prevalence, awareness, treatment and control of hypertension in the middle-aged population of China, 1992-1998. Hypertens Res 2004; 27: 703-709.

11 Lee HS, Park YM, Kwon HS, Lee JH, Park YJ, Lim SY, Lee SH, Yoon KH, Son HY, Kim DS, Yim HW, Lee WC. Prevalence, awareness, treatment, and control of hypertension among people over 40 years old in a rural area of South Korea: the Chungju Metabolic Disease Cohort (CMC) Study. Clin Exp Hypertens 2010; 32: 166-178.

12 Leelacharas S. Hypertension in Thailand. Prog Cardiovasc Nurs 2009; 24: 196-198.

13 Costanzo S, Di Castelnuovo A, Zito F, Krogh V, Siani A, Arnout J, Cappuccio FP, Miller MA, van Dongen M, de Lorgeril M, de Gaetano G, Donati MB, lacoviello L. Prevalence, awareness, treatment and control of hypertension in healthy unrelated male-female pairs of European regions: the dietary habit profile in European communities with different risk of myocardial infarction - the impact of migration as a model of geneenvironment interaction project. J Hypertens 2008; 26: 2303-2311.

14 Agyemang C, Valkengoed IV, Koopmans R, Stronks K. Factors associated with hypertension awareness, treatment and control among ethnic groups in Amsterdam, The Netherlands: the SUNSET study. J Hum Hypertens 2006; 20: 874-881.

15 Wu Y, Tai ES, Heng D, Tan CE, Low LP, Lee J. Risk factors associated with hypertension awareness, treatment, and control in a multi-ethnic Asian population. J Hypertens 2009; 27: 190-197.

16 Chen R, Tunstall-Pedoe H, Morrison C, Connaghan J, A'Brook R. Trends and social factors in blood pressure control in Scottish MONICA surveys 1986-1995: the rule of halves revisited. J Hum Hypertens 2003; 17: 751-759.

17 Stamler R, Stamler J, Riedlinger W, Algera G, Roberts R. Family (parental) history and prevalence of hypertension: results of a nationwide screening program. JAMA 1979; 241: 43-46.

18 Degli Esposti E, Di Martino M, Sturani A, Russo P, Dradi C, Falcinelli S, Buda S. Risk factors for uncontrolled hypertension in Italy. J Hum Hypertens 2004; 18: 207-213.

19 The sixth report of the Joint National Committee on prevention, detection, evaluation, and treatment of high blood pressure. Arch Intern Med 1997; 157: 2413-2446.

20 Guidelines Subcommittee. 1999 World Health Organization-International Society of Hypertension guidelines for the management of hypertension. J Hypertens 1999; 17: 151-184. 